2018

\title{
Towards Dynamic Interaction-Based Reputation Models
}

\author{
Almas Melnikov \\ Innopolis University \\ Manuel Mazzara \\ Innopolis University \\ Victor Rivera \\ Innopolis University
}

See next page for additional authors

Follow this and additional works at: https://arrow.tudublin.ie/scschcomart

Part of the Artificial Intelligence and Robotics Commons

\section{Recommended Citation}

Almaz Melnikov, JooYoung Lee, Victor Rivera, Manuel Mazzara, Luca Longo: Towards Dynamic Interaction-Based Reputation Models. IEEE 32nd International Conference on Advanced Information Networking and Applications, AINA 2018, pp.422-428 DOI: 10.1109/AINA.2018.00070

This Conference Paper is brought to you for free and open access by the School of Computer Sciences at ARROW@TU Dublin. It has been accepted for inclusion in Articles by an authorized administrator of ARROW@TU Dublin. For more information, please contact arrow.admin@tudublin.ie, aisling.coyne@tudublin.ie, gerard.connolly@tudublin.ie.

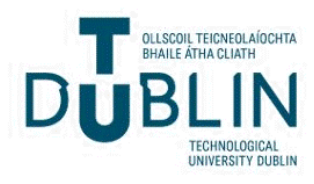




\section{Authors}

Almas Melnikov, Manuel Mazzara, Victor Rivera, JooYoung Lee, and Luca Longo

This conference paper is available at ARROW@TU Dublin: https://arrow.tudublin.ie/scschcomart/123 
See discussions, stats, and author profiles for this publication at: https://www.researchgate.net/publication/327003300

\section{Towards Dynamic Interaction-Based Reputation Models}

Conference Paper · May 2018

DOI: 10.1109/AINA.2018.00070

\section{CITATIONS}

5 authors, including:

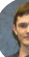

Almaz Melnikov

Innopolis University

3 PUBLICATIONS 9 CITATIONS

SEE PROFILE

Victor Rivera

Innopolis University

59 PUBLICATIONS 249 CITATIONS

SEE PROFILE

Some of the authors of this publication are also working on these related projects:

Project Jolie Good Buildings View project

Project Hikester - The Event Management Application View project
READS

149

(2) JooYoung Lee

64 PUBLICATIONS 424 CITATIONS

SEE PROFILE

Manuel Mazzara

Innopolis University

328 PUBLICATIONS 2,837 CITATIONS

SEE PROFILE 


\section{Towards dynamic interaction-based reputation models}

\author{
Almaz Melnikov, JooYoung Lee, Manuel Mazzara, Victor Rivera \\ Innopolis University \\ Russian Federation, Tatarstan republic, Innopolis 420500 \\ Emails: a.melnikov@innopolis.ru,j.lee@innopolis.ru,m.mazzara@innopolis.ru, \\ v.rivera@innopolis.ru
}

\author{
Luca Longo \\ School of Computing \\ Dublin Institute of Technology, \\ Dublin, Republic of Ireland \\ Email: luca.longo@dit.ie
}

\begin{abstract}
In this paper, we investigate how dynamic properties of reputation can influence the quality of users' ranking. Reputation systems should be based on rules that can guarantee high level of trust and help identify unreliable units. To understand the effectiveness of dynamic properties in the evaluation of reputation, we propose our own model (DIB-RM) that utilizes three factors: forgetting, cumulative, and activity period. In order to evaluate the model, we use data from StackOverflow which also has its own reputation model. We estimate similarity of ratings between DIB-RM and the StackOverflow reputation model to test our hypothesis. We use two values to calculate our metrics: DIB-RM reputation and historical reputation. We found out that historical reputation gives better metric values. Our preliminary results are presented for different sets of values of the aforementioned factors in order to analyze how effectively the model can be used for modeling reputation systems.
\end{abstract}

\section{INTRODUCTION}

A feature of interaction-based Internet communities is that direct connections and relationships between users do not have a significant influence on assessing their reputations. Rather, the most influential aspect for such an assessment is the behavior and the activities of the users within a digital community. The computation of user reputation and the assessment of user rating are directly connected because reputation is used for comparing users and at the same time rating is based upon that comparison. Rating systems are used in Internet communities where people communicate with each other, share opinions, information as well as find new contacts. One type of Internet communities includes web-sites where questioning and answering (Q\&A) is possible, e.g. Ask.fm and Yahoo! answers allow users to ask questions on a wide range of topics. Other examples include platforms such as StackOverflow $(\mathrm{SO})$ that focuses on more specialized topics such as Computer Science. Q\&A sites are built upon the notion of community contributions. Here, users generate content by asking specific questions to the community. In turn, other users of the same community can answer them, thus generating peer-reviewed content. The quality of this content depends mainly on the human expertise and knowledge. Hence an open problem is how to assess the level of expertise of those users. StackOverflow has its own model for the assessment of the reputation. It is mainly based upon a voting mechanism that allows users to recommend (like) or disapprove (dislike) the quality of questions or answers. This mechanism helps to determine the expertise and reputation of each user within the community. Here, reputation is an integer value from zero to infinity. As a consequence, users can be ordered and compared by this reputation value.

This study is focused on the investigation of how dynamic factors - factors that add dynamism to reputation - can be successfully used for rating users. The hypothesis is that dynamic aspects such as past activity, cumulative past knowledge and forgetting (inactivity) can be meaningfully used in computing the reputation of users as well as their trustworthiness in interaction-based Internet communities. This hypothesis is exploited with the data generated by the StackOverflow platform.

The paper is organized as follows: section II describes related works on reputation and trust. Section III focuses on the design of a novel model of reputation, called DIBRM, that employs dynamic factors. Section IV evaluates DIBRM highlighting the impacts of the dynamic factors on the assessment of reputation. Finally, Section V is devoted to conclusion pf the study and future work.

\section{RELATED WORK}

\section{A. Reputation and trust}

Trust can be defined by person's positive or negative expectations of another person's actions. Reputation is a collective measure of trustworthiness based on the referrals or ratings from members of a community. In [19], authors systematize knowledge about trust and reputation. They highlight the problem that many researchers use these terms as equal and therefore, they explain and separate them. Authors propose the schema depicted in Fig. 1 which shows the hierarchy of trust types. Reputation is a type of trust called "Global trust". The first level in the classification is based on the number of people who participate in trust evaluation:

1) Local trust - trust which exists between two people.

2) Global trust - trust is the result of deposing of the many users' opinions towards a particular user.

Another separation is performed by a method of collecting information:

1) Explicit - the value is directly given by users. 
2) Implicit - the value is based on users' activity and interaction, according to available data and made assumptions.

The concept of trust has been investigated thoroughly, and several properties have been defined: context-specific, $d y$ namic, transitive, asymmetric, direction. As mentioned in [19], reputation has only three of them:

- Context-specific. Reputation can be different between the same units of a system in a different scope. Rousseau discussed this specific nature of trust in social and psychological sciences [20]. In [10], authors extracted reputation of email authors based on the contents of the email as well as the forward/reply behaviors. Also, reputation management in vehicular networks is discussed widely to overcome pitfalls of security systems [6].

- Dynamic. Chang E. describes this property in a way that reputation changes on time perspective continuously [2]. Also, new interactions have more influence on a reputation value because they are more relevant and important than old ones. A lot of techniques have been invented and they implement this concept [8], [24], [12], [25].

- Transitive. This is the most common property which is widely used in several models. The reputation of a person depends on indirect connections of other people. There are several examples [21], [18].

Non-commercial trust-based platforms have been proposed in the past [17]. However, temporal factors have been rarely used as an exclusive factor in the computation of trust.

\section{B. Reputation models}

Internet communities such as social networks, e.g. Facebook, MySpace or Twitter, grow in size (number of users) daily. Users of such social networks have many problems related to trust. For example, a user needs to know a level of trustworthiness of a service provider or a product supplier before making a choice, or evaluate a new person before accepting his/her request [5]. Due to the growth of social networks, researchers give their attention to trust and reputation management problems. Measurement of trust in social networks is based on several principles. Wanita Sherchan separates reputation models into three groups [22]:

1) Network Structure/Graph-Based models.

2) Interaction-Based models.

3) Hybrid models.

This separation is based on the type of technique which is used in the model. Models with a network structure use the concept of "Web of trust" or FOAF (Friend-Of-A-Friend). This concept uses "Transitivity" property and direct connections among people to evaluate the trust value between two people. Kutter and Golbeck [9] came out with their model for calculating inference trust in social networks which are called SUNNY. Jiang and Wang [7] proposed SWTrust algorithm, it generates a small graph from a big online social network (OSN). Authors in [4] presented a model which provides a movie

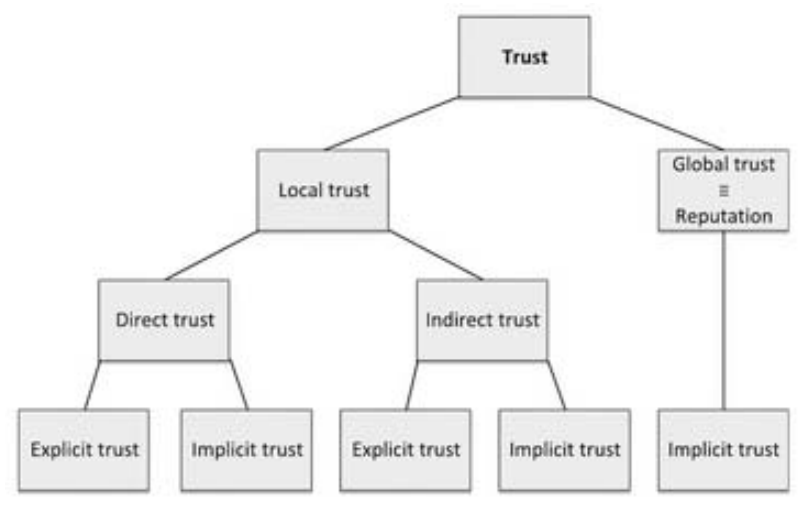

Figure 1: Taxonomy of trust

recommendation and it is based on an average score of users' ratings of films. However, this type of models does not take into account interactions between members. The activity of users and the nature of their communications particularly affect the trust or reputation value.

Some trust models consider only interactions between system nodes, in contrast to graph-based interaction previously mentioned [11]. Such models are called interaction-based models. Liu et al. actively use in [14] interactions between users in online platforms for predicting trust value. They take into account two groups of parameters: metrics of user's activity with data such as frequency of reviews and ratings and taxonomy of different connections between two users. Kamvar et al. [8] propose the EigenTrust algorithm which performs reputation evaluation on history and state of interactions with the system. It uses aging to differentiate importance of new interactions and old ones. Hybrid models combine graph structure of system and interactions between the units of that system. Anupam et. al provide the "SecuredTrust" model [3] which evaluates trust between multi-agent system units for load balancing and finding malicious agents. This model accounts for a historical information that does not allow malicious units to change their trust value in a short period of time. They also implement decreasing of trust value of previous interactions that increases the influence of current activity of the unit.

Lee et. al combines the topological information (position of each node in a given network) as well as interactions between nodes [13]. They proposed a reputation computation model, called ReMSA, considers feedbacks, voting and time decaying to update reputation everytime when there is a new interaction. The voting mechanism is recursive so that a node can collect feedbacks (about the target node) from remote nodes who are not directly connected to the given node.

Longo et al. [15] check the hypothesis that temporal based factors, such as activity, frequency, regularity and presence, can be used as an evidence of an entity's trustworthiness. They introduce a new algorithm and provide tests on Wikipedia database that is composed of 12000 users and 94000 articles. 
They compared prediction metrics with Wikipedia ratings and had satisfactory results. Good prediction rate was $60 \%$, bad prediction rate was less than $20 \%$, so this approach can be useful in trust measurement and can be aggregated with more traditional methods. The main drawback of using temporal factors is the amount of information required. A lot of data is needed to evaluate the trustworthiness of articles and compare them to each other, because interactions are distributed on time interval where the article exists. The same author proposed a methodology to continuously align a trust model in force with the changing context within dynamic applications such as forums, blogs, $\mathrm{p} 2 \mathrm{p}$ systems. The self-adaptation is reflected in the auto-organisation of the trust function aimed at assessing an agents' trustworthiness [16].

Adali et al. evaluated trust in a social network, which is based on interaction behavior between two users and a propagation of messages of each other [1]. The first feature is called conversation trust, the second propagative trust. These trust metrics depend only on the communication traffic stream, therefore models are interaction-based ones. Only information about the sender, receiver and time parameters of messages were used. Authors investigated the relevance of using these features on Twitter social network database. They divide messages into several sets by proximity of time. These sets are called conversations. Long conversations are also more confidently balanced conversations. Propagative trust is higher if users share messages to third parties.

Several models were designed for trust and reputation evaluation. They solve different problems from implementing recommendation system to reaching the high quality of service and system load balancing.

\section{Research question and hypothesis}

Some researchers improve models by making them more complex and heavier in computation to achieve better results. On another hand, some of them try to create more simple models without significant decrease of results but with better performance. We select the second approach. So, if reputation model based on interactions will give satisfactory results, implementing a reputation system which needs to store additional data and requires creating and managing new logic is redundant. The research question of this paper is: To what extent a model, built upon dynamic interaction factors, can approximate subjective voting of users within the StackOverflow community?

\section{DyNAMIC INTERACTION BASEd REPUTATION MOdEL}

Trust can be seen as the amount of interaction among people: the more interaction occurs between two individuals the more one of them trust the other. This makes trust very unstable, it actually changes continuously over time [23]. We introduce Dynamic Interaction Based Reputation Model (DIBRM), a model that captures this dynamic property of trust.

DIB-RM is an interaction-based model among users of a community over time. The model computes a reputation value for each user of the system combining different factors: forgetting factor, the continuous decrease of reputation of an individual; cumulative factor, the importance of users' activities; and activity period factor, the period of time in which the change in the reputation value happened.

DIB-RM updates the reputation value of each interaction using a fixed number of parameters. This removes the need for storing information about previous interactions. Also, it works in dynamic environments. Thus a model can update the reputation value of the users while they provide some actions.

The following sections explain the assumptions made by the model, the mathematical background for DIB-RM and the metrics used to test the hypothesis.

\section{A. Trust Properties}

DIB-RM is built upon the following two properties of trust behavior:

1) if two individuals have no interactions for a long period of time, the trust level between them starts to decrease;

2) if two individuals interact very frequently and regularly, the trust level between them should increase faster than when they communicate rarely.

The first property is based on the dynamic property of trust. It requires the continuous change of trust levels over time. The second property comes from [23]. Authors use the "fragile trust" concept to represent that trust levels can change rapidly during short period of time depending on the activity of the user.

\section{B. Model description}

In Internet Communities, interactions occur when there is an activity between two individuals. As an example, in StackOverflow there is an interaction between a user and the system when a user posts a question, or between users when a user answers an already posted question.

Interactions in DIB-RM are modeled by $I_{n}$

$$
I_{n}=I_{b_{n}}+I_{c_{n}}
$$

where $n \in 0 \ldots N$ is the index of the interaction and $N$ is the total number of interactions of a user. $I_{n}$ contains a time stamp, when the interaction takes place, and a value that describes the contribution to the reputation. They can be enumerated by time stamp to form historical chain of user's activity.

Interactions have different effects to the trust value. Each interaction has a basic value $I_{b_{n}}$. Depending on the state of communication between a user and the system characterized by activity and frequency, an interaction can be perceived differently. $I_{c_{n}}$ capture the cumulative part of the interaction, the second property of trust held by DIB-RM. It is defined as:

$$
I_{c_{n}}=I_{b_{n}} * \alpha *\left(1-\frac{1}{A_{n}+1}\right)
$$

where $\alpha$ is the weight of the cumulative part. It shows how big $I_{c_{n}}$ can grow (if $\alpha=1$ then $I_{c_{n}} \in 0 \ldots I_{b_{n}}$ ). $A_{n}$ is the number of sequential activity periods.

Figure 2 depicts the dependency of interaction values from a number of activity periods for different weights of $\alpha$ and 


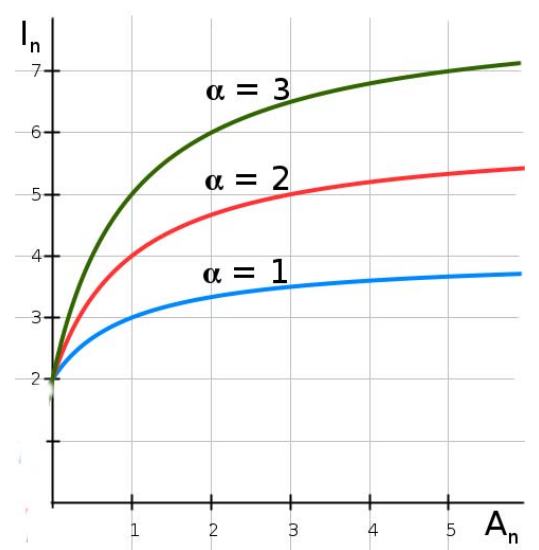

Figure 2: Interaction value graph for different $\alpha$, weight of "cumulative" effect.

$I_{b}=2$. So for $\alpha=1 I_{c}$ can be maximum 2 , for $\alpha=2$ maximum 4 , for $\alpha=3$ - maximum 6 .

Social communities have different contexts and features that affect the properties of the system. One of these properties is the frequency of user communication which is defined as the period of time between the last two activities. DIB-RM models this property as $t_{a}$. As an example, $t_{a}$ for Wikipedia can be one week, when a user creates or edits some article whereas for StackOverflow it can be one day, when user answers to a question.

$$
\Delta_{n}=\left[\frac{t_{n}-t_{n-1}}{t_{a}}\right]
$$

is the number of periods between the 2 last interactions. If the difference between $t_{n}$ and $t_{n-1}$ is less than $t_{a}$ the number of activity periods will increase by one. It means, the user continues to communicate frequently.

The final formula for trust is

$$
T_{n}=T_{n-1} * \beta^{\Delta_{n}}+I_{n}, \beta \in[0,1]
$$

where $\beta$ is the forgetting factor that is chosen by each system individually. If $\beta$ is close to 1 , the trust value decreases.

Also, if save DIB-RM reputation values of a user for each day and represent results as a graph, it will look like the line which is depicted in Fig. 3. Another parameter which can be calculated is the sum of previous reputation values. This parameter is close to a value of an area which is under the graph line. We also use it to compare DIB-RM and StackOverflow model because it accumulates historical information about a user's reputation. Even if a user currently has low reputation value but was very active before and done a lot of operations, the sum can be high in comparison with other users. We call this parameter historical reputation.

In order to achieve objective results, several components should be presented. On one hand, it is a reputation model, on another, it is data which will be used for evaluations.

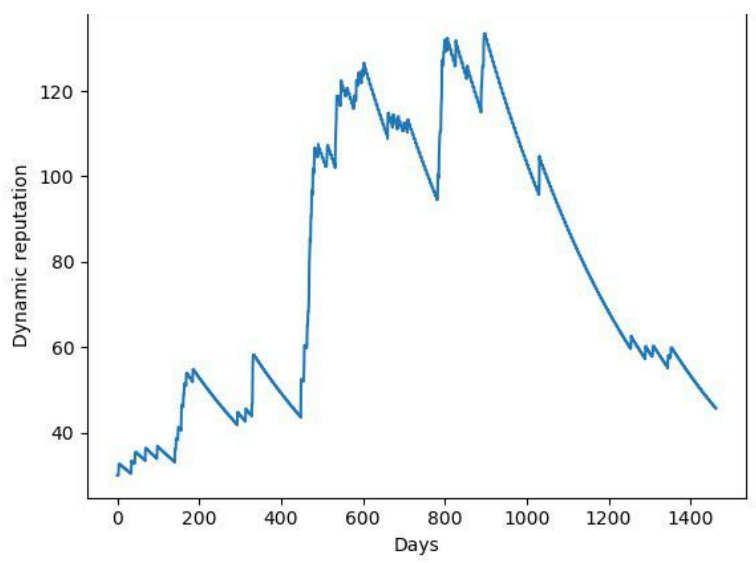

Figure 3: DIB-RM user reputation graph

\section{Metric of approximation}

Reputation values mean nothing in isolation. It is a relative value used for comparison of users. In general, if the reputation value of user $A$ is higher than the reputation of user $B$, the trustworthiness of user $A$ is also higher.

To measure the efficiency of DIB-RM, we applied the model StackOverflow and compared the results to StackOverflow's own rating system. The results of this comparison will give information about how DIB-RM approximates StackOverflow voting system. The metric is defined as

$$
\mu_{D}=1-\frac{1}{N^{2}} * \sum_{i=1}^{N}\left(\frac{1}{D} * \sum_{j=1}^{D}\left|R_{S_{i j}}-R_{D_{i j}}\right|\right)
$$

where $N$ is the number of users, $D$ the number of days between first and last dates, $R_{S_{i j}}$ the StackOverflow reputation value of user $i$ on day $j$ and $R_{D_{i j}}$ is the DIB-RM reputation value of user $i$ on day $j .\left|R_{S_{i j}}-R_{D_{i j}}\right|$ is the absolute difference between rating places of individual $i$ on particular day $j$. This value shows how close DIB-RM rating is to StackOverflow. Then we calculate the average difference of ratings for user $i \frac{1}{D} * \sum_{j=1}^{D}\left|R_{S_{i j}}-R_{D_{i j}}\right|$ in all-days period. It allows to avoid focusing on one estimation and analyze general behavior of the model. After that, the average difference of all users is estimated. The last step is subtracting from 1 the average difference, which divided to the number of rating places $N$, gives information about how DIB-RM rating system is close to StackOverflow's one.

Another approach is measuring rating of users by historical reputation value. The formula of metric remains the same but instead of $R_{D_{i j}}$ (reputation rating place of user $i$ on day $j$ ) $R_{H_{i j}}$ (historical reputation rating) is used.

$$
\mu_{H}=1-\frac{1}{N^{2}} * \sum_{i=1}^{N}\left(\frac{1}{D} * \sum_{j=1}^{D}\left|R_{S_{i j}}-R_{H_{i j}}\right|\right)
$$

Moreover, error of metric should be estimated to have clear picture of DIB-RM work. If the model has a small error, it gives expected results. Error estimation is performed by 
calculating standard deviation of metric, $\mu$. For reputation it is $\sigma_{D}$, for historical reputation it is $\sigma_{H}$.

\section{EVAluATION AND Discussion}

We used StackOverflow to evaluate our model. StackOverflow defines its own users' reputation system. We used DIB$\mathrm{RM}$ to evaluate the reputation of users based on interactions. Then, we calculated their difference using the metrics defined in the previous section. The StackOverflow database is available online and can be downloaded from an open access repository. This resource provides $x \mathrm{ml}$ dumps for all needed files about posts, posts' history, posts' links, comments, users, votes, badges among others. For the computation of DIB$\mathrm{RM}$ and StackOverflow reputations we need posts, comments, users and votes. This is because other entities contain only details about interactions, for example, a post's history stores texts of questions and answers. This dataset includes history of user's activity from September $15^{\text {th }}$ 2008, launch day of StackOverflow, to September $14^{\text {th }} 2012$.

\section{A. Analysis of used data}

We implemented DIB-RM and its internal structure is shown in Fig. 4. Information, which is contained in $x \mathrm{ml}$ files, can be represented in a form of a table. Therefore, we performed conversion from $x \mathrm{ml}$ to $c s v$ format because it can be managed by programing tools that we used for creating the DIB-RM model. We wrote a parser which was optimized to generate output results. It operates only with required fields without converting all file to $c s v$.

The internal structure of the data from csv files provides DIB-RM with fast access to information of interactions. By interactions we consider both posts and comments because they show activity of a user and his contribution to the system. Post is a general concept of content which users produce. It can be of two types: question or answer. In this paper we do not distinguish types of interactions and assign the same reputation value to them. A typical post tuple is $<$ PostId, CreationDate, PostTypeId, ParentId,UserId $>$, a typical tuple of comment is $<$ CommentId, CreationDate, UserId $>$.

1) PostId, CommentId - positive integer which represent unique identifier of entity.

2) CreationDate - date and time when post or comment was created.

3) UserId - positive integer which represents unique identifier of the user who is the author.

Those two tuples have similar domains, so we can store them together. Sorting of interactions dataset by (UserId,CreationDate) key pair will give historical sequence for each user. We do not add votes as interactions to DIB-RM because the purpose is to compare it with StackOverflow model which is based on a voting system.

Vote entities are required to make simulation of StackOverflow model. We created a program which is fully based on rules of calculating users' reputation

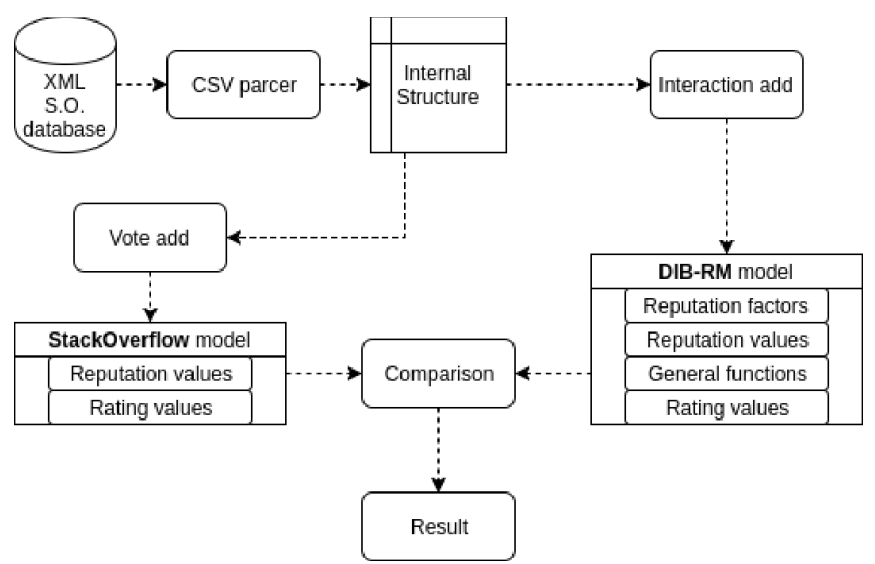

Figure 4: DIB-RM structure.

in StackOverflow. Votes' tuple has the structure < VoteId, CreatinDate, VoteTypeId, PostId, UserId >.

1) VoteId - positive integer which represent a unique identifier of vote.

2) PostId - positive integer which represent a unique identifier of post. Vote is related to this post.

3) VoteTypeId - positive integer which represent type of vote. It can have a value in the range from 0 to 9 .

4) CreationDate - date and time when vote was created.

Each post has a $U \operatorname{ser} I d$ attribute and we can connect a vote with its recipient and change his reputation.

The total amount of users that we used for the computation is 15.000 . Between the minimum and maximum StackOverflow's reputations we identified 10 equal intervals and extracted 1.500 users from each group. This method enables us to have a representative set of users. For 4 years those users generated 8.630.000 posts, 16.067.000 comments and received 33.269.000 votes.

\section{B. Activity period factor}

The first step of our experimentation is to understand the importance of the $t_{a}$ constant (activity period) essential for computing value of the "cumulative" part of interaction. We performed a set of computations changing the $t_{a}$ constant (in days) obtaining ratings' difference averages and standard deviation values shown in tables I and II. DIB-RM model has three factors that can be changed: $t_{a}, \alpha$ (cumulative factor), $\beta$ (forgetting factor). Computations are provided with fixed $\alpha=1$ and $\beta=0.99$ for both parameters reputation and historical reputation.

The results which are provided in tables I and II show that if $t_{a}$ (activity period) increases, the metric value also increases for both parameters. It comes from the nature of the StackOverflow model which calculates reputation by adding value of a new vote to the sum of previous ones and does not decrease over time. So, when $t_{a}$ increases, the reputation value starts to decrease after a longer period of time, users have wider window to interact and increase cumulative part of interactions' reputation value. That means reputation keeps 
Table I: Table of reputation metric results for different $t_{a}$ values

\begin{tabular}{|l|l|l|l|}
\hline$\#$ & $t_{a}$ & $\mu_{D}$ & $\sigma_{D}$ \\
\hline 1 & 1 & 0,8122 & 0,1100 \\
\hline 2 & 2 & 0,8313 & 0,0936 \\
\hline 3 & 4 & 0,8510 & 0,0744 \\
\hline 4 & 8 & 0,8605 & 0,0604 \\
\hline
\end{tabular}

Table II: Table of historical reputation metric results for different $t_{a}$ values

\begin{tabular}{|l|l|l|l|}
\hline$\#$ & $t_{a}$ & $\mu_{H}$ & $\sigma_{H}$ \\
\hline 1 & 1 & 0,8816 & 0,0128 \\
\hline 2 & 2 & 0,8805 & 0,0026 \\
\hline 3 & 4 & 0,8813 & 0,0086 \\
\hline 4 & 8 & 0,8808 & 0,0021 \\
\hline
\end{tabular}

almost the same or increases to the high value of interaction because the cumulative part also decreases less often. It makes reputation lose a dynamic property and become static as the StackOverflow model.

Historical reputation does not decrease as StackOverflow reputation and it also contains values of all interactions. That is why historical reputation approximates better than the StackOverflow's model reputation value. Values of metric in table II are between 0.88 and 0.882 . That means increasing of $t_{a}$ does not have significant influence on metric results.

Four graphs are depicted in Fig. 5 for different $t_{a}$ (activity period) values. They show reputation changes for two users over time. The red line belongs to user with $i d=300$, the blue line to user with $i d=235$. Comparison of these lines shows that high value of $t_{a}$ increases the distance between reputation values. At first sub-graph user 235 has four times greater reputation than user 300 . In one period of time in the range from 800 'th day to 1100 'th day, the blue line is higher than the red line. However, on the fourth sub-graph, where $t_{a}$ parameter equals to 8 , the blue line has higher value just at the beginning.

\section{Forgetting factor}

In this section we analyze the influence of forgetting factor to metric results and to reputation value. Forgetting factor is used to decrease the importance of previous interactions, so new ones have more influence on a reputation. We use two forgetting factor values $\beta=0.99$ and $\beta=0.9$ that means a reputation reduces to $1 \%$ or to $10 \%$ for each activity period. Hence a combination of forgetting factor and activity period factor is also important. The results of computations are presented in tables III and IV.

We provide metric values for four cases where $\alpha$ is fixed and equals to $1, t_{a}$ has two variants, 2 and 8 , and $\beta$ equals to 0.99 and 0.90 . Increasing the forgetting factor leads to raising

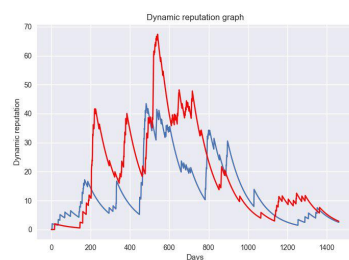

(a) $t_{a}=1$

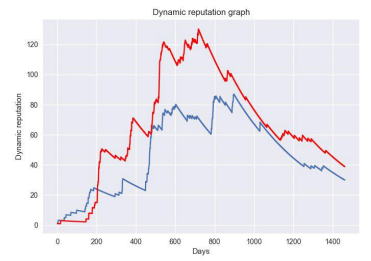

(c) $t_{a}=4$

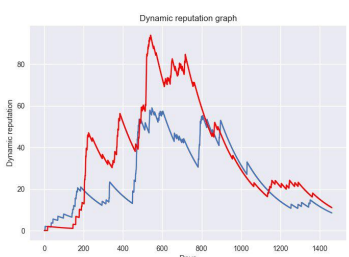

(b) $t_{a}=2$

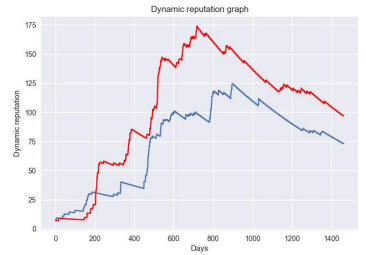

(d) $t_{a}=8$
Figure 5: Dynamic reputation graph for different $t_{a}$

of the metric value that means previous interactions' values are also important for reputation evaluation. If rating of users is built on DIB-RM reputation, changing a $\beta$ value has significant influence on the metric. In case of $t_{a}=2 \mu_{D}$ grows from 0.79 to 0.83 when $t_{a}=8 \mu_{D}$ grows from 0.81 to 0.86 .

Table III: Table of reputation metric results for different $\beta$ values

\begin{tabular}{|l|l|l|l|l|}
\hline$\#$ & $t_{a}$ & $\beta$ & $\mu_{D}$ & $\sigma_{D}$ \\
\hline 1 & 2 & 0.90 & 0,7900 & 0,1285 \\
\hline 2 & 2 & 0.99 & 0,8313 & 0,0936 \\
\hline 3 & 8 & 0.90 & 0,8193 & 0,0992 \\
\hline 4 & 8 & 0.99 & 0,8605 & 0,0604 \\
\hline
\end{tabular}

Table IV: Table of historical reputation metric results for different $\beta$ values

\begin{tabular}{|l|l|l|l|l|}
\hline$\#$ & $t_{a}$ & $\beta$ & $\mu_{H}$ & $\sigma_{H}$ \\
\hline 1 & 2 & 0.90 & 0,8803 & 0,0024 \\
\hline 2 & 2 & 0.99 & 0,8805 & 0,0026 \\
\hline 3 & 8 & 0.90 & 0,8808 & 0,0023 \\
\hline 4 & 8 & 0.99 & 0,8808 & 0,0021 \\
\hline
\end{tabular}

\section{Cumulative factor}

Cumulative factor $\alpha$ represents the proportion of basic part and cumulative part of interaction. Cumulative part directly depends on the activity of a user. If a user sequentially performs interactions that have an interval between each other less than the activity period, the value of cumulative part increases. We provide evaluation for four cases when $t_{a}=2$, $\beta=0.99, \alpha=\{1,2,4,8\}$. The result values are shown in tables $\mathrm{V}$ and VI. 
Table V: Table of reputation metric results for different $\alpha$ values

\begin{tabular}{|l|l|l|l|}
\hline$\#$ & $\alpha$ & $\mu_{D}$ & $\sigma_{D}$ \\
\hline 1 & 1 & 0,8313 & 0,0936 \\
\hline 2 & 2 & 0,8441 & 0,0774 \\
\hline 3 & 4 & 0,8426 & 0,0814 \\
\hline 4 & 8 & 0,8515 & 0,0723 \\
\hline
\end{tabular}

Table VI: Table of historical reputation metric results for different $\alpha$ values

\begin{tabular}{|l|l|l|l|}
\hline$\#$ & $\alpha$ & $\mu_{H}$ & $\sigma_{H}$ \\
\hline 1 & 1 & 0,8805 & 0,0026 \\
\hline 2 & 2 & 0,8806 & 0,0025 \\
\hline 3 & 4 & 0,8808 & 0,0030 \\
\hline 4 & 8 & 0,8808 & 0,0023 \\
\hline
\end{tabular}

\section{CONCLusion AND Future Work}

In this paper, we investigated the usage of dynamic factors for reputation evaluation. We formally defined reputation model, DIB-RM, which combines all factors: forgetting factor, cumulative factor and active period factor. Our evaluation was performed in the context of StackOverflow web site. Results are based on a 4-year history, covering 15.000 users, more than 8.000.000 posts and 33.000.000 votes. We tested our factors and hypothesis by comparing ratings of users that are created by DIB-RM and StackOverflow model. We used two values for creating ratings: reputation and historical reputation. Historical reputation value gave better results, around $88 \%$ similarity between DIB-RM and StackOverflow ratings. Results of evaluation show that this value is resistant to factors' changes, so it allows to adopt the model to various environments by selecting different values of factors without decreasing of metric value. We believe that these factors can be used as an evidence of users' trustworthiness in combination with more traditional ones. Our further works will be addressed to determining environments in whose context dynamic factors can be used as a strong evidence of trustworthiness.

\section{REFERENCES}

[1] Sibel Adali, Robert Escriva, Mark K Goldberg, Mykola Hayvanovych, Malik Magdon-Ismail, Boleslaw K Szymanski, William A Wallace, and Gregory Williams. Measuring behavioral trust in social networks. In Intelligence and Security Informatics (ISI), 2010 IEEE International Conference on, pages 150-152. IEEE, 2010.

[2] Elizabeth Chang, Patricia Thomson, Tharam Dillon, and Farookh Hussain. The fuzzy and dynamic nature of trust. Trust, privacy, and security in digital business, pages 161-174, 2005.

[3] Anupam Das and Mohammad Mahfuzul Islam. Securedtrust: a dynamic trust computation model for secured communication in multiagent systems. IEEE Transactions on Dependable and Secure Computing, 9(2):261-274, 2012.

[4] Jennifer Golbeck. Generating predictive movie recommendations from trust in social networks. Trust Management, pages 93-104, 2006.
[5] Sana Hamdi. Computational models of trust and reputation in online social networks. PhD thesis, Université Paris-Saclay, 2016.

[6] Rasheed Hussain, Waqas Nawaz, JooYoung Lee, Junggab Son, and Jung Taek Seo. A hybrid trust management framework for vehicular social networks. In Hien T. Nguyen and Vaclav Snasel, editors, Computational Social Networks, pages 214-225, Cham, 2016. Springer International Publishing.

[7] Wenjun Jiang and Guojun Wang. Swtrust: Generating trusted graph for trust evaluation in online social networks. In Trust, Security and Privacy in Computing and Communications (TrustCom), 2011 IEEE 10th International Conference on, pages 320-327. IEEE, 2011.

[8] Sepandar D Kamvar, Mario T Schlosser, and Hector Garcia-Molina. The eigentrust algorithm for reputation management in $\mathrm{p} 2 \mathrm{p}$ networks. In Proceedings of the 12th international conference on World Wide Web, pages 640-651. ACM, 2003.

[9] Ugur Kuter and Jennifer Golbeck. Sunny: A new algorithm for trust inference in social networks using probabilistic confidence models. In AAAI, volume 7, pages 1377-1382, 2007.

[10] JooYoung Lee, Yue Duan, Jae C. Oh, Wenliang Du, Howard Blair, Lusha Wang, and Xing Jin. Social network based reputation computation and document classification. J. UCS, 18(4):532-553, 2012.

[11] JooYoung Lee, Kontantin Lopatin, Rasheed Hussain, and Waqas Nawaz. Evolution of friendship: A case study of mobiclique. In Proceedings of the Computing Frontiers Conference, CF'17, pages 267-270, New York, NY, USA, 2017. ACM.

[12] JooYoung Lee and J. C. Oh. Convergence of true cooperations in bayesian reputation game. In 2014 IEEE 13th International Conference on Trust, Security and Privacy in Computing and Communications, pages 487-494, Sept 2014.

[13] JooYoung Lee and Jae C. Oh. A Node-Centric Reputation Computation Algorithm on Online Social Networks, pages 1-22. Springer International Publishing, Cham, 2015.

[14] Haifeng Liu, Ee-Peng Lim, Hady W Lauw, Minh-Tam Le, Aixin Sun, Jaideep Srivastava, and Young Kim. Predicting trusts among users of online communities: an epinions case study. In Proceedings of the 9th ACM conference on Electronic commerce, pages 310-319. ACM, 2008.

[15] Luca Longo, Pierpaolo Dondio, and Stephen Barrett. Temporal factors to evaluate trustworthiness of virtual identities. In Security and Privacy in Communications Networks and the Workshops, 2007. SecureComm 2007. Third International Conference on, pages 11-19. IEEE, 2007.

[16] Luca Longo, Pierpaolo Dondio, Bresciani Riccardo, Andrew Butterfield, and Stephen Barrett. Enabling adaptation in trust computations. Computationworld, 2009.

[17] Manuel Mazzara, Luca Biselli, Pier Paolo Greco, Nicola Dragoni, Antonio Marraffa, Nafees Qamar, and Simona de Nicola. Social networks and collective intelligence: a return to the agora. IGI Global, 2013.

[18] Lik Mui, Mojdeh Mohtashemi, and Ari Halberstadt. A computational model of trust and reputation. In System Sciences, 2002. HICSS. Proceedings of the 35th Annual Hawaii International Conference on, pages 2431-2439. IEEE, 2002.

[19] Monika Rakoczy, Amel Bouzeghoub, Katarzyna Wegrzyn-Wolska, and Alda Gancarski Lopes. Users views on others-analysis of confused relation-based terms in social network. In OTM Confederated International Conferences" On the Move to Meaningful Internet Systems", pages 155-174. Springer, 2016.

[20] Denise M Rousseau, Sim B Sitkin, Ronald S Burt, and Colin Camerer. Not so different after all: A cross-discipline view of trust. Academy of management review, 23(3):393-404, 1998.

[21] Jordi Sabater and Carles Sierra. Reputation and social network analysis in multi-agent systems. In Proceedings of the first international joint conference on Autonomous agents and multiagent systems: Part 1, pages 475-482. ACM, 2002.

[22] Wanita Sherchan, Surya Nepal, and Cecile Paris. A survey of trust in social networks. ACM Comput. Surv., 45(4):47:1-47:33, August 2013.

[23] Megan Tschannen-Moran and Wayne K Hoy. A multidisciplinary analysis of the nature, meaning, and measurement of trust. Review of Educational Research, 70(4):547-593, 2000.

[24] Yanchao Zhang and Yuguang Fang. A fine-grained reputation system for reliable service selection in peer-to-peer networks. IEEE Transactions on Parallel and Distributed Systems, 18(8), 2007.

[25] Runfang Zhou and Kai Hwang. Powertrust: A robust and scalable reputation system for trusted peer-to-peer computing. IEEE Transactions on parallel and distributed systems, 18(4), 2007. 